\title{
Lossy Gossip and Composition of Metrics
}

\author{
Andries E. Brouwer • Jan Draisma • Bart J. Frenk
}

Received: 23 May 2014 / Revised: 9 January 2015 / Accepted: 15 January 2015 /

Published online: 24 February 2015

(C) The Author(s) 2015. This article is published with open access at Springerlink.com

\begin{abstract}
We study the monoid generated by $n \times n$ distance matrices under tropical (or min-plus) multiplication. Using the tropical geometry of the orthogonal group, we prove that this monoid is a finite polyhedral fan of dimension $\left(\begin{array}{l}n \\ 2\end{array}\right)$, and we compute the structure of this fan for $n$ up to 5. The monoid captures gossip among $n$ gossipers over lossy phone lines, and contains the gossip monoid over ordinary phone lines as a submonoid. We prove several new results about this submonoid as well. In particular, we establish a sharp bound on chains of calls in each of which someone learns something new.
\end{abstract}

Keywords Min-plus matrix multiplication · Finite metrics · Gossip · Tropical algebraic groups

\section{Introduction and Results}

Imagine travelling between three locations such as Eindhoven ( $E$, a medium-sized town in the Netherlands), a parking lot $P$ on the border of the Dutch capital Amsterdam, and the city centre $A$ of Amsterdam. In Fig. 1, the travel times by car between these locations are depicted by the leftmost triangle, while the travel times by bike are

Editor in charge: Günter M. Ziegler

A. E. Brouwer · J. Draisma · B. J. Frenk

Department of Mathematics and Computer Science, Technische Universiteit Eindhoven, P.O. Box 513, 5600 MB Eindhoven, The Netherlands e-mail: aeb@cwi.nl

J. Draisma

e-mail: j.draisma@tue.nl

B. J. Frenk

e-mail: b.j.frenk@tue.nl 

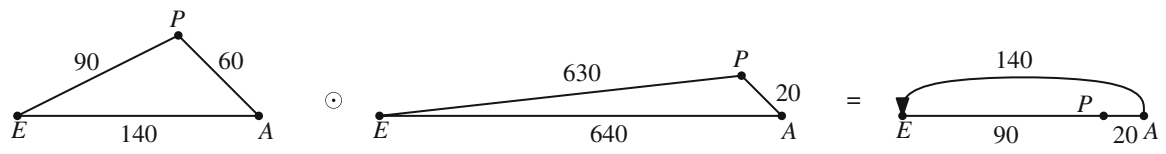

Fig. 1 Composing the car metric with the bike metric

depicted by the second triangle. The large distances between $E$ and either $P$ or $A$ are covered much faster by car than by bike. On the other hand, because of crowded streets, the short distance between $P$ and $A$ is covered considerably faster by bike than by car. As a consequence, an attractive alternative for travelling from $E$ to $A$ by car is to travel by car from $E$ to $P$ and continue by bike to $A$. In other words, to get from $E$ to $A$ we first do a step in the car metric and then a step in the bike metric, where we optimise the sum of the two travel times. Computing this car-bike metric for the remaining ordered pairs leads to the picture on the right in Fig. 1. The corresponding matrix computation is

$$
\left[\begin{array}{ccc}
0 & 90 & 140 \\
90 & 0 & 60 \\
140 & 60 & 0
\end{array}\right] \odot\left[\begin{array}{ccc}
0 & 630 & 640 \\
630 & 0 & 20 \\
640 & 20 & 0
\end{array}\right]=\left[\begin{array}{ccc}
0 & 90 & 110 \\
90 & 0 & 20 \\
140 & 20 & 0
\end{array}\right],
$$

where $\odot$ is tropical or min-plus matrix multiplication, obtained from usual matrix multiplication by changing plus into minimum and times into plus. Note that the resulting matrix is not symmetric (the transpose corresponds to the "first bike, then car" metric), and that it does not satisfy the triangle inequality either. The bike metric and the car metric were both picked from the 3-dimensional cone of symmetric matrices satisfying all triangle inequalities. Hence one might think that such tropical products sweep out a $3+3=6$-dimensional set. However, if we perturb the travel times in the two metric matrices slightly, then their min-plus product moves only in a threedimensional space, where the entry at position $(1,3)$ remains the sum of the entries in positions $(1,2)$ and $(2,3)$, while the entry in position $(3,1)$ moves freely. This preservation of dimension when tropically multiplying cones of distance matrices is one of the key results of this paper.

While keeping this min-plus product in the back of our minds, we next contemplate the following different setting. Each of the three gossipers, Eve, Patricia, and Adam, has an individual piece of gossip, which they can share through one-to-one phone calls in which both callers update each other on all the gossip they know. Record the knowledge of $E, P, A$ in a three-by-three uncertainty matrix with entries 0 (for " $i$ 's gossip is known by $j$ ") and $\infty$ (for the other entries). Then initially that matrix is the tropical identity matrix, with zeroes along the diagonal and $\infty$ outside the diagonal. A phone call between $E$ and $P$, for example, corresponds to tropically right-multiplying that tropical identity matrix with

$$
\left[\begin{array}{ccc}
0 & 0 & \infty \\
0 & 0 & \infty \\
\infty & \infty & 0
\end{array}\right]
$$

resulting in this very same matrix. A second phone call between $P$ and $A$ leads to 


$$
\left[\begin{array}{ccc}
0 & 0 & \infty \\
0 & 0 & \infty \\
\infty & \infty & 0
\end{array}\right] \odot\left[\begin{array}{ccc}
0 & \infty & \infty \\
\infty & 0 & 0 \\
\infty & 0 & 0
\end{array}\right]=\left[\begin{array}{ccc}
0 & 0 & 0 \\
0 & 0 & 0 \\
\infty & 0 & 0
\end{array}\right]
$$

Note the resemblance of this computation with the car-bike metric computation above. This resemblance can be made more explicit by passing from gossip to lossy gossip, where each phone call between gossipers $k$ and $l$ comes with a parameter $q \in[0,1]$ to be interpreted as the fraction of information that gets broadcast correctly through the phone line, and where each gossiper $j$ knows a fraction $p_{i j} \in[0,1]$ of $i$ 's gossip. Assume the (admittedly simplistic) procedure where $k$ updates his knowledge of gossip $i$ to $q \cdot p_{i l}$ if this is larger than $p_{i k}$ and retains his knowledge $p_{i k}$ of gossip $i$ otherwise, and similarly for gossiper $l$. In this manner, the fractions $p_{i j}$ are updated through a series of lossy phone-calls. Passing from $p_{i j}$ to the uncertainty $u_{i j}:=-\log p_{i j} \in$ $[0, \infty]$ of gossiper $j$ about gossip $i$ and from $q$ to the loss $a:=-\log q \in[0, \infty]$ of the phone line in the call between $k$ and $l$, the update rule changes $u_{i k}$ into the minimum of $u_{i k}$ and $u_{i l}+a$, and similarly for $u_{i l}$. This is just tropical right-multiplication with the matrix $C_{k l}(a)$ having 0's on the diagonal, $\infty$ 's everywhere else, except an $a$ on positions $(k, l)$ and $(l, k)$. So lossy gossip is tropical matrix multiplication. Note that lossy gossip is different from gossip over faulty telephone lines discussed in [2,11], and also from gossip algorithms via multiplication of doubly stochastic matrices as in [5] (though the elementary matrices $W_{k l}$ there are reminiscent of our matrices $C_{k l}$ ).

This paper concerns the entirety of such uncertainty matrices, or compositions of finite metrics. Our main result uses the following notation: fixing a number $n$ (of gossipers or vertices); let $D=D_{n}$ be the set of all metric $n \times n$ matrices, i.e. matrices with entries $a_{i j} \in \mathbb{R}_{\geq 0}$ satisfying $a_{i i}=0$ and $a_{i j}=a_{j i}$ and $a_{i j}+a_{j k} \geq a_{i k}$. For standard notions in polyhedral geometry, we refer to [20].

Throughout the paper, we give $[0, \infty]$ the topology of the one-point compactification of $[0, \infty)$, i.e. the topology of a compact, closed interval.

Theorem 1.1 The set $\left\{A_{1} \odot \cdots \odot A_{k} \mid k \in \mathbb{N}, A_{1}, \ldots, A_{k} \in D_{n}\right\}$ is the support of a (finite) polyhedral fan of dimension $\left(\begin{array}{l}n \\ 2\end{array}\right)$, whose topological closure in $[0, \infty]^{n \times n}$ (with product topology) is the monoid generated by the matrices $C_{k l}(a)$ with $k, l \in[n]:=$ $\{1, \ldots, n\}$ and $a \in[0, \infty]$.

We will denote that monoid by $G_{n}$, and call it the lossy gossip monoid with $n$ gossipers. The most surprising part of this theorem is that the dimension of $G_{n}$ is not larger than $\left(\begin{array}{l}n \\ 2\end{array}\right)$. We will establish this in Sect. 7 by proving that $G_{n}$ is contained in the tropicalisation of the orthogonal group $\mathrm{O}_{n}$.

Theorem 1.2 For $n \leq 5$ the fan in the previous theorem is pure and connected in codimension 1. Moreover, for $n \leq 4$, there is a unique coarsest such fan. This coarsest fan has $D_{2}, D_{3}, D_{4}$ among the 1, 7, 289 full-dimensional cones; and in total it has 1, 2, 16 orbits of full-dimensional cones under the groups $\operatorname{Sym}(2), \operatorname{Sym}(3), \operatorname{Sym}(4)$, respectively.

For some statistics for $n=5$ we refer to Sect. 6 . We conjecture that the pureness and connectedness in codimension 1 carry through to arbitrary $n$.

About the length of products we can say the following. 
Theorem 1.3 For $n \leq 5$ every element of $G_{n}$ is the tropical product of at most $\left(\begin{array}{l}n \\ 2\end{array}\right)$ lossy phone call matrices $C_{k l}(a)$, but not every element is the tropical product offewer factors.

We conjecture that the restriction $n \leq 5$ can be omitted.

Our next result concerns "pessimal" ordinary gossip (the least efficient way to spread information, keeping the gossipers entertained for as long as possible).

Theorem 1.4 Any sequence of phone calls among $n$ gossiping parties such that in each phone call both participants exchange all they know, and at least one of the parties learns something new, has length at most $\left(\begin{array}{l}n \\ 2\end{array}\right)$, and this bound is attained.

This implies a bound on the length of irredundant products of matrices $C_{k l}(0)$, i.e. tropical products where leaving out any factor changes the value of the product.

Corollary 1.5 In the monoid generated by the matrices $C_{k l}(0), k, l \in[n]$ every irredundant product of such matrices has at most $\left(\begin{array}{l}n \\ 2\end{array}\right)$ factors.

Our motivation for this paper is twofold. First, it establishes a connection between gossip networks and composition of metrics that seems worth pursuing further. Second, the lossy gossip monoid is a beautiful example of a submonoid of $(\mathbb{R} \cup\{\infty\})^{n \times n}$; a general theory of such submonoids also seems very worthwhile. Note that subgroups of this semigroup (but with identity element an arbitrary idempotent matrix) have been investigated in [14].

The remainder of this paper is organised as follows: Sections 2 and 3 contain observations that pave the way for the analysis for $n=3,4$ in Sects. 4 and 5. In Sect. 6 we report on extensive computations for $n=5$. In Sect. 7 we discuss tropicalisations of the special linear groups and the orthogonal groups, and use the latter to prove the first statement of Theorem 1.1. Interestingly, no polyhedral-combinatorial proof of Theorem 1.1 is known. In Sect. 8 we study the monoid generated by the ordinary gossip matrices $C_{k l}(0), k, l \in[n]$ : using the ordinary orthogonal group we prove Theorem 1.4, and for $n \leq 9$ we determine the order of this monoid. We conclude with a number of open questions in Sect. 9.

\section{Preliminaries}

Fixing a natural number $n$, we define $\overline{D_{n}}$ to be the topological closure of $D_{n}$ in $[0, \infty]^{n \times n}$, and we denote by $G_{n}$ the monoid generated by $\overline{D_{n}}$ under min-plus matrix multiplication. We call $G_{n}$ the lossy gossip monoid with $n$ gossipers. This terminology is justified by the following lemma.

Lemma 2.1 The lossy gossip monoid $G_{n}$ is generated by the lossy phone call matrices $C_{k l}(a)(k, l \in[n], a \in[0, \infty])$ having zeroes on the diagonal and $\infty$ everywhere else except for values a on positions $(k, l)$ and $(l, k)$.

Proof Lossy phone call matrices lie in $\overline{D_{n}}$, so the monoid that they generate is contained in $G_{n}$. For the converse it suffices to show that every element $A$ of $\overline{D_{n}}$ is the 
product of lossy phone call matrices. We claim that, in fact, $A=\prod_{k<l} C_{k l}\left(a_{k l}\right)=: B$, where the $a_{k l}$ are the entries of $A$ and the product is taken in any order. Indeed, the $(i, j)$-entry of $B$ is the minimum of expressions of the form $a_{i_{0}, i_{1}}+a_{i_{1}, i_{2}}+\cdots+a_{i_{s-1}, i_{s}}$ where $s \leq\left(\begin{array}{l}n \\ 2\end{array}\right), i_{0}=i, i_{s}=j$, and where the $C_{i_{0}, i_{1}}, \ldots, C_{i_{s-1}, i_{s}}$ (with $\left.s \leq\left(\begin{array}{l}n \\ 2\end{array}\right)\right)$ appear in that order (though typically interspersed with other factors) in the product expression for $B$. By the triangle inequalities among the entries of $A$, the minimum of these expressions equals $a_{i, j}$.

Although elements of $G_{n}$ need not be symmetric, they have a symmetric core.

Lemma 2.2 Each element $A$ of $G_{n}$ satisfies $a_{i j}=a_{j i}$ for at least $n-1$ pairs of distinct indices $i, j$. The graph with vertex set $[n]$ and these pairs as edges is connected.

Proof We need to prove that for any partition of $[n]$ into two nonempty parts $K$ and $L$ there exist a $k \in K$ and an $l \in L$ such that $a_{k l}=a_{l k}$. Write $A=C_{i_{1}, j_{1}}\left(b_{1}\right) \odot \cdots \odot$ $C_{i_{s}, j_{s}}\left(b_{s}\right)$ with $b_{1}, \ldots, b_{s} \in \mathbb{R}_{\geq 0}$. If there is no $r$ such that $i_{r}$ and $j_{r}$ lie in different sides of the partition, then $a_{k l}=a_{l k}=\infty$ for all $k \in K$ and $l \in L$. Otherwise, among all $r$ for which $i_{r}$ and $j_{r}$ lie in different parts of the partition, choose one for which $b_{r}$ is minimal. Then $a_{i_{r}, j_{r}}=a_{j_{r}, i_{r}}=b_{r}$.

Lemma 2.3 Every connected graph on $[n]$ occurs as symmetric core of some element of $G_{n}$.

Proof Number the edges of that subgraph $I_{1}, \ldots, I_{m}$ and consider the tropical product

$$
\begin{aligned}
A= & C_{I_{1}}\left(1+2^{-1}\right) \odot C_{I_{2}}\left(1+2^{-2}\right) \odot \cdots \odot C_{I_{m}}\left(1+2^{-m}\right) \\
& \odot C_{I_{1}}\left(2^{1}\right) \odot C_{I_{2}}\left(2^{2}\right) \odot \cdots \odot C_{I_{m}}\left(2^{m}\right) \\
& \odot C_{I_{1}}\left(2^{m+1}\right) \odot \cdots \odot C_{I_{m}}\left(2^{2 m}\right) \\
& \odot C_{I_{1}}\left(2^{2 m+1}\right) \odot \cdots,
\end{aligned}
$$

where the product stabilises once all edges have acquired a finite length. For $\{k, l\}$ equal to some $I_{i}$ we have $a_{k l}=a_{l k}=1+2^{-i}$, where we use that the sum of two of these $m$ numbers is larger than any third. For any other $\{k, l\}$ the value $a_{k l}$ is a sum of some number $m$ of distinct negative powers of 2, the integer $m$ itself, and some number of distinct positive powers of 2 . This sum uniquely determines the sequence of factors contributing to it, of which there are at least two. Hence the sum determines the ordered pair $(k, l)$. In particular, we have $a_{l k} \neq a_{k l}$.

Observe that $C_{k l}(a) \odot C_{k l}(b)=C_{k l}(a \oplus b)$, where $\oplus$ denotes tropical addition defined by $a \oplus b=\min (a, b)$. Thus Lemma 2.1 exhibits $G_{n}$ as a monoid generated by certain one-parameter submonoids, reminiscent of the generation of algebraic groups by one-parameter subgroups. This resemblance will be exploited in Sects. 7 and 8.

We define the length of an element $X$ of $G_{n}$ as the minimal number of factors in any expression of $X$ as a tropical product of lossy phone call matrices $C_{k l}(a)$. A rather crude but uniform upper bound on the length of elements of $G_{n}$ is the maximal number of factors in a tropical product of lossy phone call matrices in which no factor can be left out without changing the result. We call such an expression irredundant, and we have the following bounds. 
Lemma 2.4 The number of factors in any irredundant tropical product of lossy phone call matrices in $G_{n}$ is at most $n^{2}(n-1) / 2$. In particular, the length of every element of $G_{n}$ is bounded by this number.

Proof Let $A$ be an element of $G_{n}$ and write

$$
A=C_{I_{1}}\left(a_{1}\right) \odot \cdots \odot C_{I_{k}}\left(a_{k}\right),
$$

where $a_{j}$ are non-negative real numbers and $I_{j}$ are unordered pairs of distinct numbers in $[n]$. The entry at position $(h, i)$ of $A$, if not equal to $\infty$, is the minimum of expressions $a_{k_{1}}+\cdots+a_{k_{s}}$, where $\left(I_{k_{1}}, \ldots, I_{k_{s}}\right)$ is a path from $h$ to $i$ in the complete graph on $[n]$ and $k_{1}<\cdots<k_{s}$. Choose such a path with $s$ minimal, and call this the minimal path from $h$ to $i$. Since it is never cheaper to visit a vertex twice, we have $s \leq n-1$. This shows that for each of the $n(n-1)$ pairs $(h, i)$ only at most $(n-1)$ of the factors are necessary, and this gives an upper bound of $n(n-1)^{2}$ on the quantity in the lemma. The sharper bound in the lemma comes from the fact that if $j \neq h, i$ lies on a minimal path from $h$ to $i$, then $i$ does not lie on a minimal path from $h$ to $j$. Hence the total number of ordered pairs $(i, j)$ with $i, j$ unequal to $h$ and $j$ on the minimal path from $h$ to $i$ is at most $(n-1)+\left(\begin{array}{c}n-1 \\ 2\end{array}\right)=\left(\begin{array}{l}n \\ 2\end{array}\right)$, and this bounds the number of factors essential for the $h$-th row of $A$. This gives the bound.

Lemma 2.5 There exists an expression that is an irredundant tropical product of $\left(\begin{array}{c}n+1 \\ 3\end{array}\right)$ lossy phone call matrices in $G_{n}$.

Proof We proceed by induction on $n$. For $n=1$ there are no factors. Let $W_{n-1}$ be an irredundant expression over $G_{n}$ of length $\left(\begin{array}{l}n \\ 3\end{array}\right)$ not involving the index 1 . Let $P_{h}$ be the product

$$
P_{h}=C_{12}\left(b_{h 1}\right) \odot C_{23}\left(b_{h 2}\right) \odot \cdots \odot C_{h, h+1}\left(b_{h h}\right)
$$

(of length $h$ ) and put

$$
W_{n}=W_{n-1} \odot P_{n-1} \odot P_{n-2} \odot \cdots \odot P_{1} .
$$

Then the expression for $W_{n}$ has length $\left(\begin{array}{c}n+1 \\ 3\end{array}\right)$. Order the constants involved such that those in $W_{n-1}$ are small, those in $P_{1}$ (just $b_{11}$ ) much larger, those in $P_{2}$ larger again, and those in $P_{n-1}$ the largest. The matrix that is the result of multiplying out the expression $W_{n}$ has $(i, j)$-entry as found for $W_{n-1}$ when $i, j \neq 1$, but $(1, h+1)$-entry as found for $P_{h}$ (since 1 is not found in $W_{n-1}, h+1$ is not found later than in $P_{h}$, and earlier $P_{j}$ are too expensive). It follows that no factor of $P_{h}$ is redundant.

Proposition 2.6 The closure of $D_{n}$ under tropical matrix multiplication is the support of some finite polyhedral fan in $\mathbb{R}_{\geq 0}^{n \times n}$ and equals $G_{n} \cap \mathbb{R}_{\geq 0}^{n \times n}$. Its topological closure in $[0, \infty]^{n \times n}$ equals $G_{n}$.

Note that this is Theorem 1.1 minus the claim that the dimension of that fan is (not more than) $\left(\begin{array}{l}n \\ 2\end{array}\right)$; this claim will be proved in Sect. 7 . 
Proof By Lemma 2.1 and the proof of Lemma 2.4 the closure of $D_{n}$ under tropical matrix multiplication is a finite union of images of orthants $\mathbb{R}_{>0}^{k}$ with $k \leq n(n-1)^{2}$ under piecewise linear maps. Such an image is the support of some polyhedral fan. The remaining two statements are straightforward.

From now on, we will sometimes use the term "polyhedral fan" for the topological closure in $[0, \infty]^{N}$ of a polyhedral fan in $\mathbb{R}_{\geq 0}^{N}$. Thus $G_{n}$ itself is a polyhedral fan in $[0, \infty]^{n \times n}$.

Recall that the Kleene star of $A \in[0, \infty]^{n \times n}$ is defined as

$$
\begin{aligned}
A^{*} & :=I \oplus A \oplus A^{\odot 2} \oplus \cdots \\
& =I \oplus A \oplus A^{\odot 2} \oplus \cdots \oplus A^{\odot(n-1)}=(I \oplus A)^{\odot(n-1)},
\end{aligned}
$$

where $I$ is the tropical identity matrix [7, p. 21]. The $(i, j)$-entry of $A^{*}$ records the length of the shortest path from $i$ to $j$ in the directed graph on $[n]$ with edge lengths $a_{i j}$. From this interpretation it follows readily that for $A_{1}, \ldots, A_{s} \in[0, \infty]^{n \times n}$ with zero diagonal, and $\pi \in \operatorname{Sym}(s)$, we have $\left(A_{1} \odot \cdots \odot A_{s}\right)^{*}=\left(A_{\pi(1)} \odot \cdots \odot A_{\pi(s)}\right)^{*}$.

Lemma 2.7 The Kleene star maps $G_{n}$ into its subset $\overline{D_{n}}$.

Proof Let $A \in G_{n}$ be the tropical product of lossy phone call matrices $C_{1}, \ldots, C_{k}$. Note that $C_{i}^{\mathrm{T}}=C_{i}$. We have

$$
\begin{aligned}
A^{*} & =\left(C_{1} \odot \cdots \odot C_{k}\right)^{*}=\left(C_{k} \odot \cdots \odot C_{1}\right)^{*}=\left(C_{k}^{\mathrm{T}} \odot \cdots \odot C_{1}^{\mathrm{T}}\right)^{*} \\
& =\left(\left(C_{1} \odot \cdots \odot C_{k}\right)^{\mathrm{T}}\right)^{*}=\left(\left(C_{1} \odot \cdots \odot C_{k}\right)^{*}\right)^{\mathrm{T}}=\left(A^{*}\right)^{\mathrm{T}},
\end{aligned}
$$

where we have used the remark above, the fact that transposition reverses multiplication order, and the fact that Kleene star commutes with transposition. Thus $A^{*}$ is a symmetric Kleene star and hence a metric matrix.

\section{Graphs with Detours}

In the next two sections we will visualise elements of the lossy gossip monoids $G_{3}$ and $G_{4}$, as well as the polyhedral structures on these monoids. We will do this through combinatorial gadgets that we dub graphs with detours. We first recall realisations of ordinary metrics, i.e. elements of $D_{n}$ (see, e.g. $[8,13]$ ).

Let $\Gamma=(V, E)$ be a finite, undirected graph and $w: E \rightarrow \mathbb{R}_{\geq 0}$ be a function assigning lengths to the edges of $\Gamma$. The weight of a path in $(\Gamma, w)$ is the sum of the weights of the individual edges in the path. A map $\ell:[n] \rightarrow V$ is called a labelling, or [n]-labelling, if we need to be precise, and the pair $(\Gamma, \ell)$ is referred to as a labelled graph, or an $[n]$-labelled graph.

A weighted $[n]$-labelled graph gives rise to a matrix $A(\Gamma, w, \ell)$ in $D_{n}$ whose entry at position $(i, j)$ is the minimal weight of a path between $\ell(i)$ and $\ell(j)$. We say that the weighted labelled graph $(\Gamma, w, \ell)$ realises the matrix $A(\Gamma, w, \ell)$. Any matrix $X \in D_{n}$ has a realisation by some weighted, $[n]$-labelled graph, e.g. the graph with vertex set $[n]$, the entries of $X$ as weights, and $\ell$ equal to the identity. However, typically more efficient realisations exist, in the following sense. A weighted, $[n]$-labelled graph 


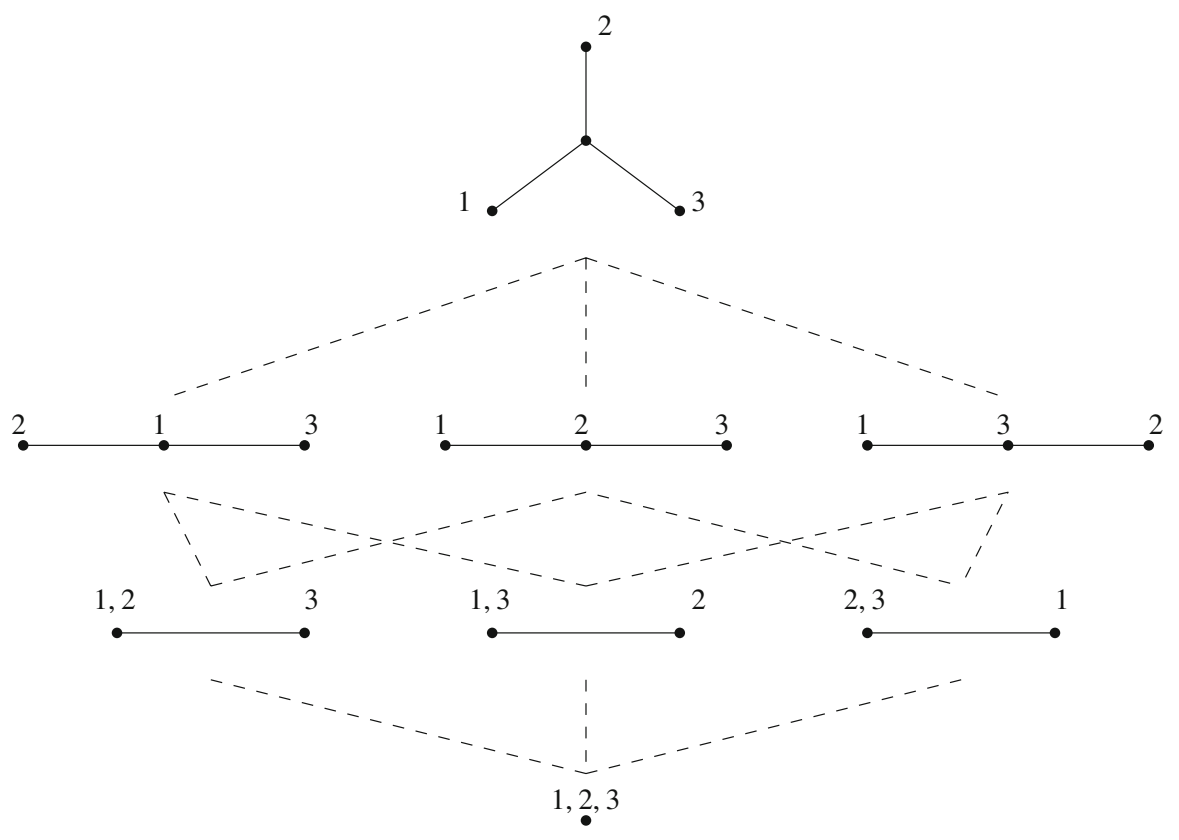

Fig. 2 Minimal realisations of three-point metrics

( $\Gamma=(V, E), w, \ell)$ is called an optimal realisation of $X$ if the sum $\sum_{e} w(e)$ is minimal among all realisations [13]. We will, moreover, require that no edges get weight 0 (since such edges can be removed and their endpoints identified), and that no vertices in $V \backslash \ell([n])$ have valency 2 (since such vertices can be removed and their incident edges glued together). Optimal realisations of any $X \in D_{n}$ exist [13], and there is an interesting question concerning the uniqueness of optimal realisations for generic $X$ [8, Conjecture 3.20].

Our first step in describing the cones of $G_{3}$ and $G_{4}$ is to find weighted labelled graphs that realise the elements of $D_{3}, D_{4}$, as follows (for much more about this see $[8,9])$. We write $J_{0}$ for the matrix of the appropriate size with all entries 0 .

Example 3.1 We give optimal realisations of the elements of $D_{n}$, for $n=2,3$, 4. For the cases $n=5,6$ see $[16,18]$.

(1) An element of $D_{2} \backslash\left\{J_{0}\right\}$ is optimally realised by the graph on two vertices having one edge with the right weight. The choice of labelling is inconsequential as long as it is injective. The matrix $J_{0}$ is optimally realised by the graph on one vertex.

(2) Any matrix in $D_{3}$ is realised by the top labelled graph of the poset depicted in Fig. 2 with suitable edge weights (note that we allow these to be zero), but only the matrices in the relative interior of the cone $D_{3}$ are optimally realised by it. Matrices on the boundary are optimally realised by some graph further down the poset, depending on the smallest face of $D_{3}$ in which the matrix lies.

(3) The case of $D_{4}$ is similar to that of $D_{3}$ in the sense that there exists a single graph $\Gamma$ which, appropriately labelled and weighted, realises any $X \in D_{4}$. However, unlike 

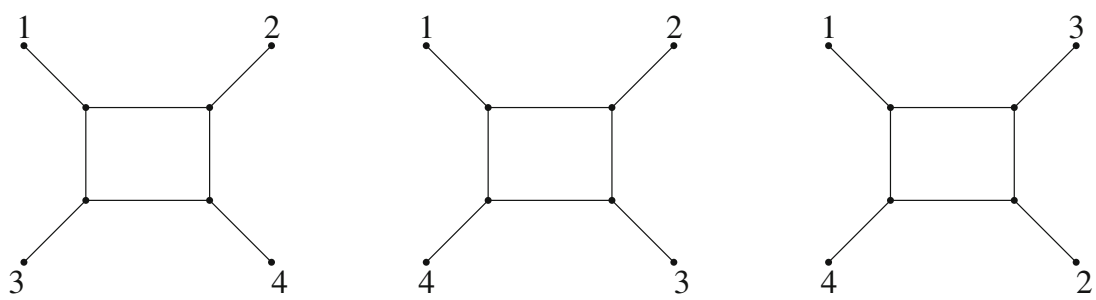

Fig. 3 Minimal realisations of four-point metrics. The parallel sides of the middle rectangle have equal weight

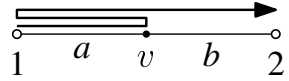

(a)

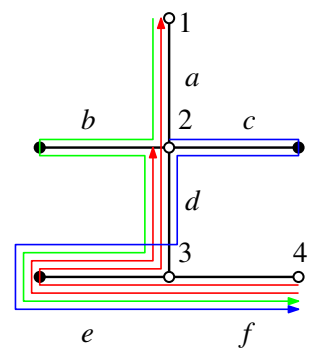

(b)

Fig. 4 Examples of labelled weighted graphs with detours. (a) Path with a single detour from 1 to 2. (b) Graph with 4 detours

for $D_{3}$, three distinct labellings are required. The labelled graphs are depicted in Fig. 3. For graphs in the relative interior of $D_{4}$, the given realisation is optimal (and in fact the unique optimal realisation).

We now extend realisation of metric matrices by graphs to realisations of arbitrary matrices in $\mathbb{R}_{\geq 0}^{n \times n}$ with zeroes on the diagonal. For this we need an extension of the concept of a labelled weighted graph. Let $i$ and $j$ be the distinct elements of $[n]$. A detour from $i$ to $j$ in an [n]-labelled weighted graph is simply a walk $p$ starting at $\ell(i)$ and ending at $\ell(j)$ that has larger total weight than the path of minimal weight between $\ell(i)$ and $\ell(j)$. Such a walk is allowed to traverse the same edge more than once. The data specifying the detour is the triple $(i, j, p)$. A labelled weighted graph with detours is a tuple consisting of a labelled weighted graph and a finite set of detours between distinct ordered pairs $(i, j)$.

Let $(\Gamma, w, \ell, \mathcal{D})$ be an $[n]$-labelled weighted graph with set of detours $\mathcal{D}$. It gives rise to a matrix $A(\Gamma, w, \ell, \mathcal{D})$ whose entry at position $(i, j)$ equals the weight of the detour from $i$ to $j$, if there is any, or the weight of a path of minimal weight between $i$ and $j$, if there is no detour between $i$ and $j$ in $\mathcal{D}$. In particular, $A(\Gamma, w, \ell, \mathcal{D})$ need not be symmetric, but its diagonal entries are 0 . Again, if $X \in \mathbb{R}_{\geq 0}^{n \times n}$ and $X=A(\Gamma, w, \ell, \mathcal{D})$, then $(\Gamma, w, \ell, \mathcal{D})$ is said to realise $X$. Any non-negative matrix with zeroes on the diagonal is realised by some labelled weighted graph with detours. Observe also that replacing all detours $(i, j, p)$ by the detours $\left(j, i, p^{\prime}\right)$, where $p^{\prime}$ is the opposite of $p$, corresponds to transposing the realised matrix. 
Example 3.2 We give two examples of labelled weighted graphs with detours. First, the graph in Fig. 4(a) has a single detour from 1 to 2, and realises the matrix

$$
\left[\begin{array}{cc}
0 & 3 a+b \\
a+b & 0
\end{array}\right] .
$$

Except when $a=0$, this matrix is not in $G_{2}$. The example in Fig. 4(b) is more interesting. It has detours between the ordered pairs $(1,4),(2,4),(4,1),(4,2)$. The weights $a, b, c, d, e, f$ are non-negative. By varying this six-tuple in $\mathbb{R}_{\geq 0}^{6}$ this graph with detours realises the 6-dimensional cone of all matrices of the form

$$
A=\left[\begin{array}{cccc}
0 & a & a+d & a+2 b+d+2 e+f \\
a & 0 & d & 2 c+d+2 e+f \\
a+d & d & 0 & f \\
f+2 e+d+a & f+2 e+d & f & 0
\end{array}\right]
$$

Observe that both $(1,4)$ and $(4,1)$ are detours, and their lengths are restricted by the inequality $a_{14} \geq a_{41}$ (indeed, the difference equals $2 b$ ). This 6-dimensional cone is one of the maximal cones in $G_{4}$, namely, cone $C_{10}$ in Fig. 6 . The graph with detours in Fig. 4(b) represents these inequalities in a visually attractive manner, but one also sees in one glance that the cone of all matrices of the form is simplicial: it is the image of $\mathbb{R}_{\geq 0}^{6}$ under an injective linear transformation into $\mathbb{R}_{\geq 0}^{4 \times 4}$. This motivates our choice for graphs with detours to represent cones of $G_{3}$ and, more importantly, $G_{4}$.

By Lemma 2.7, the Kleene star of a matrix $A$ in $G_{n}$ lies in $\overline{D_{n}}$. Thus it makes sense to look for a realisation of $A$ by a labelled weighted graph with detours that, when forgetting the detours, realises $A^{*}$. This is what we will do in the next two sections for $n=3$ and 4 .

\section{Three Gossipers}

Since $G_{3}$ is a pointed fan, no combinatorial information is lost by intersecting that fan with a sphere centred around the all-zero matrix. The resulting spherical polyhedral complex is depicted in Fig. 5. Detour graphs realising the maximal cones can be constructed by realising the arrows in an arbitrary manner as detours in the undirected graph. The middle cone is (the topological closure of) $D_{3}$, with its three codimensionone faces corresponding to the second layer in Fig. 2 and its three codimension-two faces corresponding to the third layer.

The computations to show that Fig. 5 gives all of $G_{3}$ are elementary and can be done by hand. We use pictorial notation and write $A(\Gamma)$ for the matrix realised by a labelled weighted graph with detours $\Gamma$. Here, instead of drawing a detour as a walk, we draw it as an arrow whose length is assumed to exceed the distance in the undirected graph. First, to prove that the matrices $A(\Gamma)$ with $\Gamma$ as in the figure are indeed in $G_{3}$ we observe that

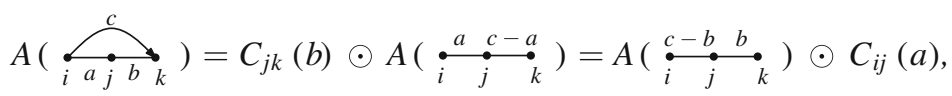




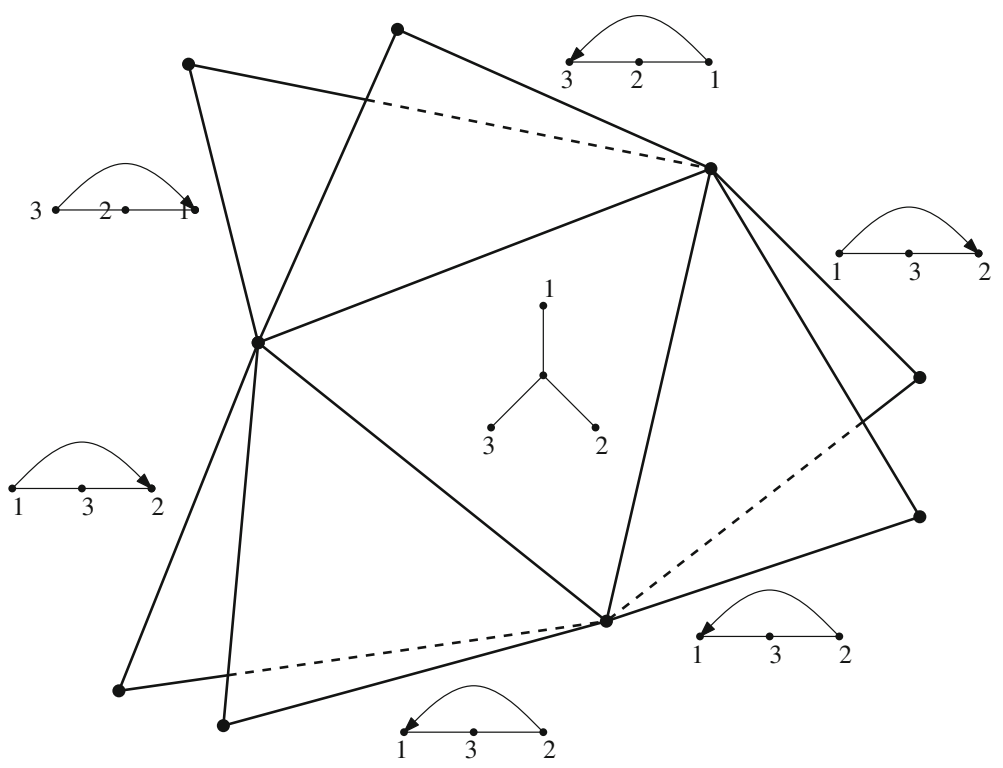

Fig. 5 Representation of the spherical complex of $G_{3}$. The labelled graphs with detours corresponding to the maximal cells are indicated. The middle triangle represents the cone of distance matrices, and on its codimension-one faces one of the three points ends up between the other two points. The remaining codimension-one faces of the remaining six cones are where one of the edge lengths in the Kleene star becomes zero

for any $c \geq a+b$ (and $a, b \geq 0$ as always). Together with the fact that $C_{i j}(a) \odot$ $C_{i j}(d)=C_{i j}(a \oplus d)$ this implies that

$$
A(\overbrace{i \underset{a_{j} b_{k}}{c}}^{c} \odot C_{i j}(d) \text { and } C_{j k}(d) \odot A(\overbrace{i a_{j} b_{k}}^{c})
$$

are contained in the complex of Fig. 5 for all choices of $a, b, c$, and $d$ with $c \geq a+b$. Next we compute

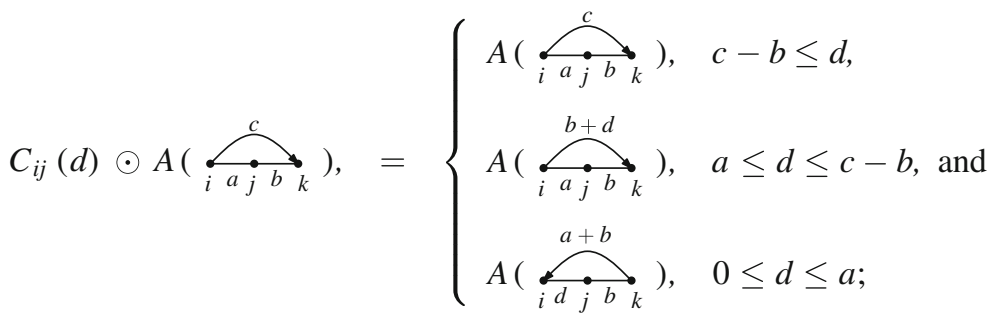

and, for $m:=\max (a-b, b-a)$, 


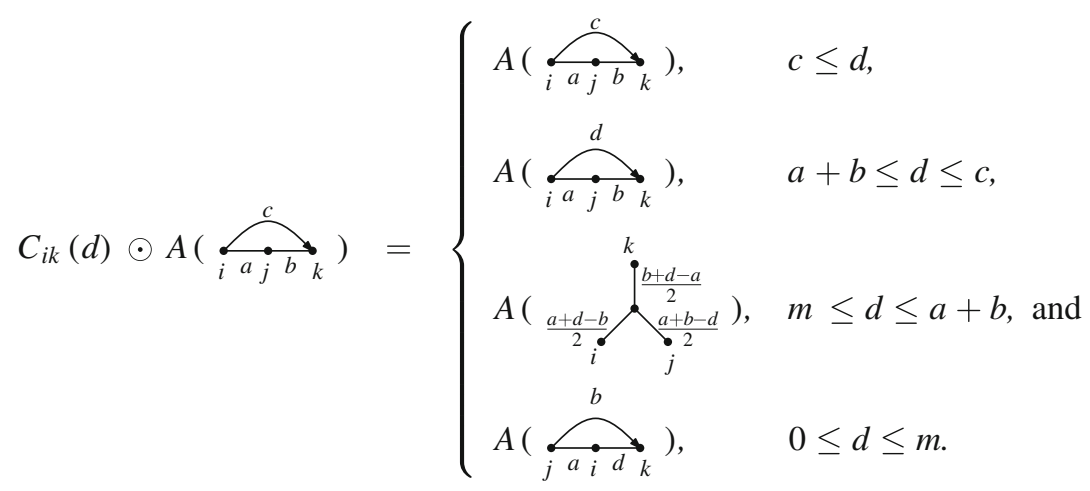

It follows by transposition that the products

$$
A(\overbrace{i a_{j} b_{k}}^{c}) \odot C_{i k}(d) \text {, and } A(\overbrace{i a_{j} b_{k}}^{c}) \odot C_{j k}(d)
$$

are also contained in one of the cones of Fig. 5. This concludes the proof of Theorem 1.2 for $n=3$.

\section{Four Gossipers}

The computations for $G_{4}$ are too cumbersome to do by hand. Instead we used Mathematica to compute a fan structure on $G_{4}$. Figure 6 gives graphs with detours realising all the cones of $G_{4}$, up to transposition and the action of Sym(4). The surplus length of a detour from $i$ to $j$ is defined as the difference between the length of the detour and the minimal distance between $i$ and $j$ in the graph. Two detours from $i$ to $j$ and from $k$ to $l$ have the same colour if their surplus lengths are equal.

These graphs were obtained as follows. First, generate all $6^{6}$ possible piecewise linear affine maps $[0, \infty]^{6} \rightarrow G_{4}$ of the form

$$
\left(a_{1}, \ldots, a_{6}\right) \rightarrow C_{I_{1}}\left(a_{1}\right) \odot C_{I_{2}}\left(a_{2}\right) \odot \cdots \odot C_{I_{6}}\left(a_{6}\right),
$$

where $I_{1}, \ldots, I_{6}$ are unordered pairs of distinct indices. Among the image cones, select only the six-dimensional ones and compute their linear spans. There are 289 different linear spans. Compute the Sym(4)-orbits on these spans; this yields 16 orbits. Choose a representative for each of these orbits on spans, and for each representative select all cones with that span. It turns out that, for each representative span, one of the cones contains all other cones. To show that the orbits of these 16 maximal cones give all of $G_{4}$, left-multiply each of these 16 cones with all possible lossy phone call matrices and show that the resulting unions of cones are contained in the union of the 289 maximal cones; this is facilitated by the fact that each of these cones is the intersection of $G_{4}$ with (the topological closure in $[0, \infty]^{n \times n}$ of) a sixdimensional subspace. Then we check that the faces of these 289 six-dimensional 


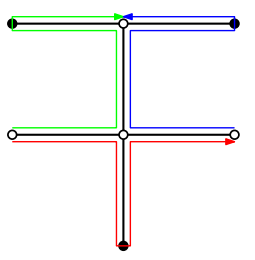

$C_{1}$

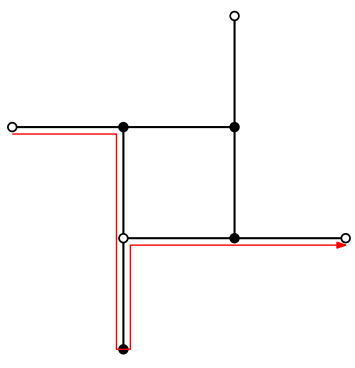

$C_{4}$

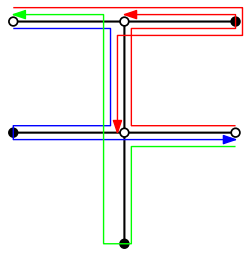

$C_{7}$

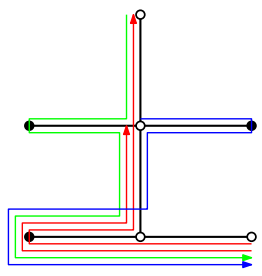

$C_{10}$

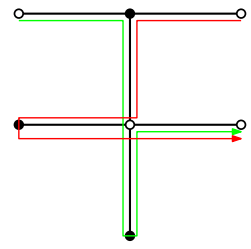

$C_{2}$

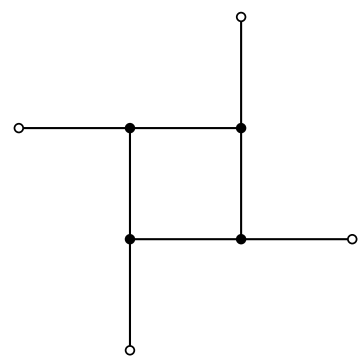

$C_{5}$
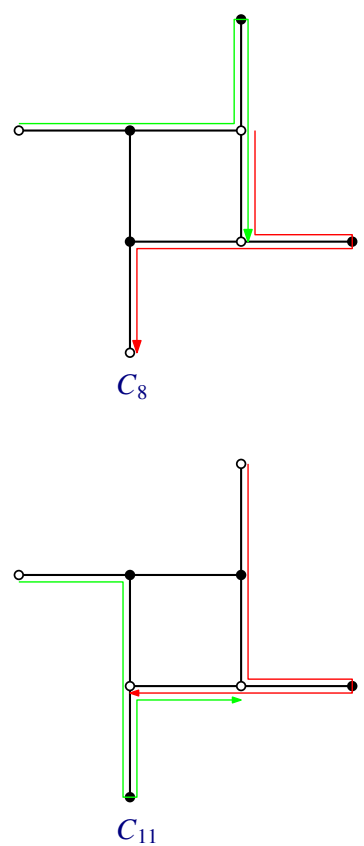

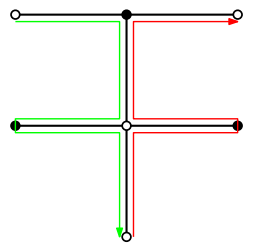

$C_{3}$

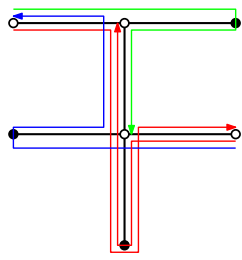

$C_{6}$

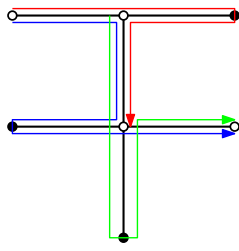

$C_{9}$

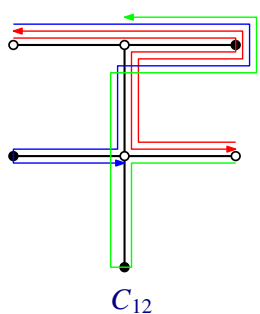

Fig. 6 Orbit representatives of labelled weighted graphs with detours realising a polyhedral fan structure on $G_{4}$ with simplicial cones. The white vertices are the labelled vertices

cones do indeed form a polyhedral fan, i.e. that the intersection of any two of these faces is a common face of both. In the process of this check, which we performed both with Mathematica and (more rapidly) with polymake [10], we find that the fan 

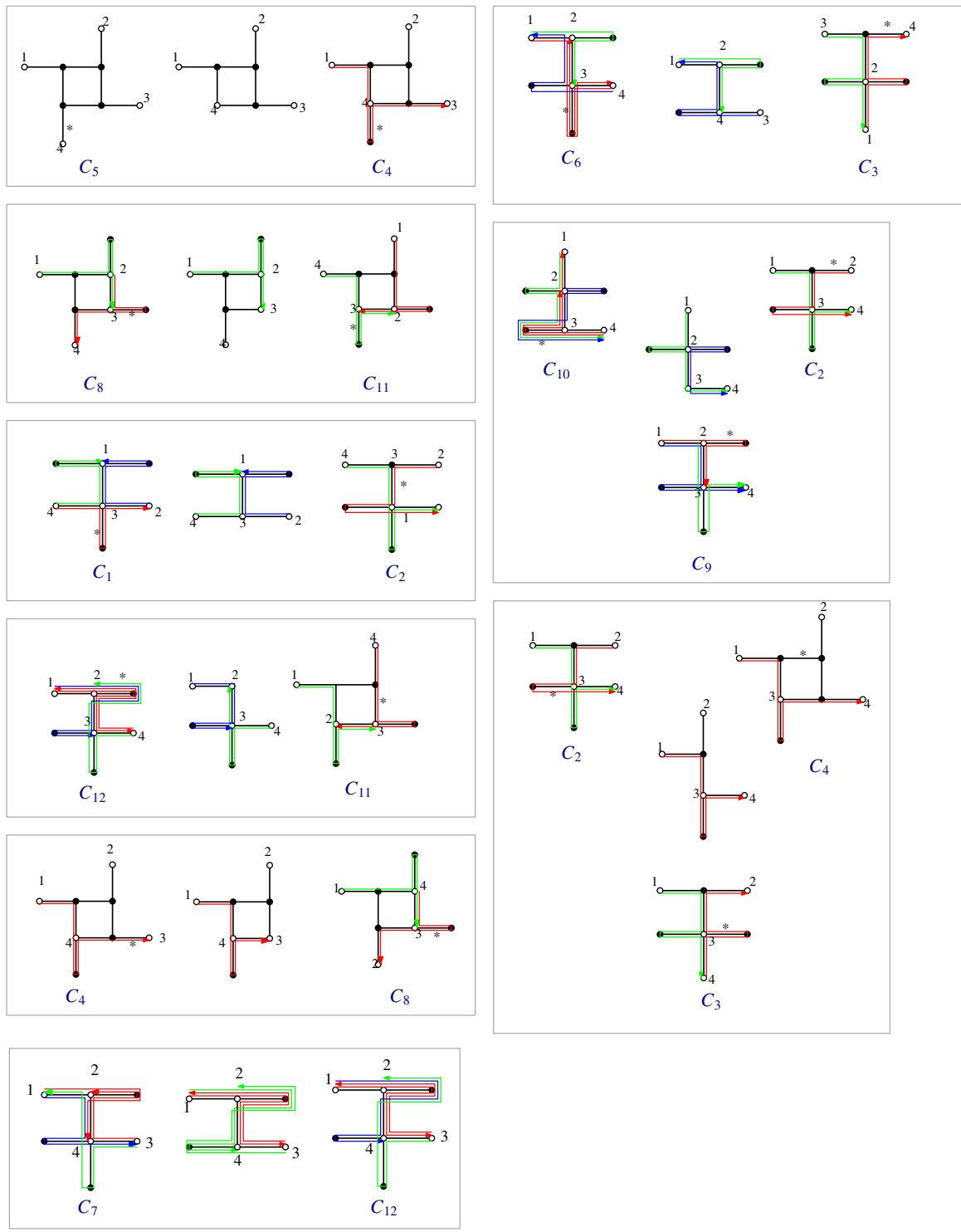

Fig. 7 Walking from maximal cones to maximal cones by edge contraction, except in case $C_{7}-C_{12}$. The edge to be contracted is indicated by an asterisk. This shows that the cones in the grey boxes intersect in a cone of dimension 5 . The intersection between $C_{7}$ and $C_{12}$ is obtained by setting equal certain surplus lengths in the graphs representing $C_{7}$ and $C_{12}$

has $f$-vector $(43,327,1042,1560,1092,289)$. This latter check yields the statement about the unique coarsest fan structure in Theorem 1.2.

Next, the group $\mathbb{Z} / 2 \mathbb{Z}$ acts on $G_{4}$ by transposition. Taking orbit representatives under the larger group $\operatorname{Sym}(4) \times(\mathbb{Z} / 2 \mathbb{Z})$ from among the 16 yields 11 cones. Among these, 9 are simplicial (have six facets), the cone $D_{4}$ has 12 facets, and the remaining 
cone has 9 facets. The cone $D_{4}$ is the union of three simplicial cones (see Fig. 3), which are permuted by $\operatorname{Sym}(4)$, so we need only one. This is $C_{5}$ in Fig. 6 . The cone with 9 facets turns out to be the union of two simplicial cones. Splitting this up yields $C_{11}$ and $C_{12}$ in the figure. It turns out that each $C_{i}$ is the image of $\mathbb{R}_{\geq 0}^{6}$ under a linear map into $\mathbb{R}_{\geq 0}^{4 \times 4}$ with non-negative integral entries with respect to the standard bases, and that these maps can be realised using weighted, labelled graphs with detours. These are the graphs in the picture. The graphs without the detours realise the Kleene star $A^{*}$ with $A \in C_{i}$.

Finally, connectivity in codimension 1 is proved by Fig. 7. It shows that any maximal cone can be connected to $D_{4}$ by passing through (relatively open) codimension-one faces; note the specified labelling. Most intersections in Fig. 7 are of a simple type, where one of the edge weights becomes zero to go from one cone to the neighbouring cone; these contracted edges are then marked with an asterisk on both sides. The only exception is the connection from $C_{7}$ to $C_{12}$. Although (suitable elements in the Sym(4)-orbits of) these cones intersect in a five-dimensional boundary cone, the boundary cone is obtained from the parameterisations specified by the graphs with detours by restricting the parameterisation to a hyperplane where two of the weights are equal. This leads to the following theorem.

Theorem 5.1 The cones realised by the graphs of Fig. 6 give a polyhedralfan structure on $G_{4}$. This polyhedral fan is pure of dimension 6 and connected in codimension 1. Its intersection with a sphere around the origin is a simplicial spherical complex. Moreover, every element of $G_{4}$ is the product of (at most) 6 lossy phone call matrices.

Remark 5.2 The spherical complex of Fig. 5 clearly has trivial homology. This phenomenon persists for $n=4$ : a computation using polymake shows that all homology groups of the intersection of $G_{4}$ with the unit sphere in 16-dimensional space are zero. We do not know whether this is true for general $n$.

\section{Five Gossipers}

More extensive computations establish the claimed facts about $G_{5}$. Since $6^{6}$ is a small number, but $10^{10}$ is not, the computation requires many refinements. We omit the details. It turns out that every element has an expression as a tropical product of at most 10 lossy phone call matrices. The set $G_{5}$ is the support of a polyhedral fan which is pure of dimension 10, and connected in codimension 1. Some statistics are given in Table 1. The single orbit of size 1 is that of $D_{n}$.

Table 1 Numbers of subspaces spanned by full-dimensional cones and their numbers of orbits under $\operatorname{Sym}(n)$

\begin{tabular}{rrrl}
\hline$n$ & \# spans & \# orbits & Orbit size distribution \\
\hline 2 & 1 & 1 & $1 \times 1$ \\
3 & 7 & 2 & $1 \times 1,1 \times 6$ \\
4 & 289 & 16 & $1 \times 1,6 \times 12,9 \times 24$ \\
5 & 91151 & 787 & $1 \times 1,2 \times 20,1 \times 30,48 \times 60,735 \times 120$ \\
\hline
\end{tabular}


The situation for $n=5$ is more complicated than that for smaller $n$ in that it no longer true that the subspace spanned by a polyhedral cone of maximal dimension intersects $G_{5}$ in a convex cone (recall that for $n=4$ this did hold, and that we used this in the proof that $G_{4}$ has a unique coarsest fan structure).

Example 6.1 Consider the open, 10-dimensional cone $P$ consisting of all matrices

$$
C_{45}(a) C_{34}(b) C_{45}(c) C_{24}(d) C_{45}(e) C_{14}(f) C_{12}(g) C_{23}(h) C_{13}(i) C_{15}(j)
$$

where we have left out the $\odot$ sign for brevity, and where the parameters $a, \ldots, j$ satisfy the inequalities

$$
\begin{aligned}
& a>c, \quad e>c, \quad f>d+g, \quad b+d>h, \quad h>g+i, \quad c+d+g+i>a+b, \\
& b+i>d+g, \quad c+d+g>j, \quad i+j>b+c, \quad c+j>d+g, \quad g+j>d+e .
\end{aligned}
$$

Similarly, consider the open, 10-dimensional cone $Q$ consisting of all matrices

$$
C_{45}(a) C_{34}(b) C_{45}(c) C_{24}(d) C_{45}(e) C_{15}(f) C_{12}(g) C_{24}(h) C_{23}(i) C_{13}(j)
$$

with inequalities

$$
\begin{aligned}
& a>c, \quad e>c, c+d+g>f, \quad c+f>d+g, \quad f+g>c+d, \\
& h>d, \quad b+d>i, i>g+j, \quad f+j>a+b, \quad b+j>d+g .
\end{aligned}
$$

In matrix form, these matrices are

$$
\left[\begin{array}{ccccc}
0 & g & i & f & j \\
g & 0 & g+i & d & d+e \\
i & h & 0 & b & b+c \\
d+g & d & b & 0 & c \\
j & c+d & a+b & c & 0
\end{array}\right] \text { and }\left[\begin{array}{ccccc}
0 & g & j & g+h & f \\
g & 0 & g+j & d & d+e \\
j & i & 0 & b & b+c \\
d+g & d & b & 0 & c \\
f & c+d & a+b & c & 0
\end{array}\right] \text {. }
$$

The linear spans of $P$ and $Q$ are the same, and the closures of $P$ and $Q$ cover all 10-dimensional cones in $G_{5}$ with this span. The latter matrix becomes the former after the substitution $f \rightarrow j, h \rightarrow f-g, i \rightarrow h, j \rightarrow i$, and this substitution turns its inequalities into

$$
\begin{aligned}
& a>c, \quad e>c, \quad c+d+g>j, \quad c+j>d+g, \quad g+j>c+d, \\
& f>d+g, \quad b+d>h, \quad h>g+i, \quad i+j>a+b, \quad b+i>d+g .
\end{aligned}
$$

We see that both cones satisfy

$a>c, e>c, \quad f>d+g, \quad b+d>h, \quad h>g+i, \quad b+i>d+g, c+j>d+g$, $c+d+g>j, \quad i+j>b+c, c+d+g+i>a+b, g+j>c+d$.

In addition, $P$ satisfies $g+j>d+e$ and $Q$ satisfies $i+j>a+b$. It follows that $P$ and $Q$ have a 10 -dimensional intersection and their union is not a convex cone. 


\section{Tropicalising Matrix Groups}

In the previous sections we have established Theorem 1.2 through explicit computations. We do not know of any systematic, combinatorial description of a polyhedral structure on $G_{n}$ for larger $n$. However, we will now establish that $G_{n}$, which is the support set of some finite polyhedral fan by Lemma 2.4, has dimension $\left(\begin{array}{l}n \\ 2\end{array}\right)$. Clearly, since $G_{n}$ contains $D_{n}$, we have $\operatorname{dim} G_{n} \geq \operatorname{dim} D_{n}=\left(\begin{array}{l}n \\ 2\end{array}\right)$. So the difficulty of Theorem 1.1 is in proving that its dimension does not exceed $\left(\begin{array}{l}n \\ 2\end{array}\right)$.

For this, we make an excursion into tropical geometry. Recall that if $K$ is a field with a non-Archimedean valuation $v: K \rightarrow \mathbb{R}_{\infty}:=\mathbb{R} \cup\{\infty\}$ and if $I \subseteq K\left[x_{1}, \ldots, x_{m}\right]$ is an ideal, then the tropical variety associated to $I$ is the set of all $w \in \mathbb{R}_{\infty}^{n}$ such that for each polynomial $f=\sum_{\alpha} c_{\alpha} x^{\alpha} \in I$ the minimum $\min _{\alpha}\left(v\left(c_{\alpha}\right)+w \cdot \alpha\right)$ is attained for at least two distinct $\alpha \in \mathbb{N}^{n}$. We denote this tropicalisation by $\operatorname{Trop}(X)$, where $X$ is the scheme over $K$ defined by $I$. For standard tropical notions we refer to [17]. If $(L, v)$ is any valued extension of $K$, then the coordinate-wise valuation map $v: L^{n} \rightarrow \mathbb{R}_{\infty}^{n}$ maps $X(L)$ into $\operatorname{Trop}(X)$. If, moreover, $v: L \rightarrow \mathbb{R}_{\infty}$ is non-trivial and $L$ is algebraically closed, then the image of the map $X(L) \rightarrow \operatorname{Trop}(X)$ is dense in $\operatorname{Trop}(X)$ in the Euclidean topology. Together with the Bieri-Groves theorem [4], this implies that the set $\operatorname{Trop}(X)$ is (the closure in $\mathbb{R}_{\infty}^{n}$ of) a polyhedral complex of dimension equal to $\operatorname{dim} X$.

We now specialise to matrix groups. As a warm-up, consider the special linear group $\mathrm{SL}_{n}$, defined over $\mathbb{Q}$ by the single polynomial $\operatorname{det}(x)-1$, where $x$ is an $n \times n$-matrix of indeterminates. Since there is only one defining polynomial and all its coefficients are \pm 1 , the valuation does not matter and $\operatorname{Trop}\left(\mathrm{SL}_{n}\right)$ equals the set of all $A=\left(a_{i j}\right)_{i j} \in$ $\mathbb{R}_{\infty}^{n \times n}$ for which the tropical determinant

$$
\operatorname{tdet}(A):=\min _{\pi \in \operatorname{Sym}(n)}\left(a_{1 \pi(1)}+\cdots+a_{n \pi(n)}\right)
$$

is either zero, or else negative and attained at least twice.

Proposition 7.1 The tropicalisation $\operatorname{Trop}\left(\mathrm{SL}_{n}\right)$ is a monoid under tropical matrix multiplication.

Proof For $A, B \in \operatorname{Trop}\left(\mathrm{SL}_{n}\right)$ set $C:=A \odot B$. A straightforward computation shows that the tropical determinant is (tropically) submultiplicative, so that $\operatorname{tdet}(C) \leq \operatorname{tdet}(A)+\operatorname{tdet}(B) \leq 0+0=0$. Hence it suffices to show that if $\operatorname{tdet}(C)<0$, then there are at least two permutations realising the minimum in the definition of $\operatorname{tdet}(C)$. Let $\pi \in \operatorname{Sym}(n)$ be one minimiser of the expression $c_{1 \pi(1)}+\cdots+c_{n \pi(n)}$. For each $i \in[n]$ let $\sigma(i) \in[n]$ be such that $c_{i \pi(i)}=a_{i \sigma(i)}+b_{\sigma(i) \pi(i)}$. Now there are two cases: either $\sigma$ is a permutation or there exist $i, j$ with $\sigma(i)=\sigma(j)$. In the latter case, also the permutation $\pi \circ(i, j) \neq \pi$ is a minimiser, and we are done. In the former case, write $\pi=\tau \circ \sigma$. Then we have

$$
\begin{aligned}
0>\operatorname{tdet}(C) & =c_{1 \pi(1)}+\cdots+c_{n \pi(n)} \\
& =\left(a_{1 \sigma(1)}+\cdots+a_{n \sigma(n)}\right)+\left(b_{1 \tau(1)}+\cdots+b_{n \tau(n)}\right),
\end{aligned}
$$


so that at least one of $\operatorname{tdet}(A)$ and $\operatorname{tdet}(B)$ is negative. If $\operatorname{tdet}(A)<0$, then since $A \in \operatorname{Trop}\left(\mathrm{SL}_{n}\right)$, there exists a permutation $\sigma^{\prime} \neq \sigma$ such that

$$
a_{1 \sigma^{\prime}(1)}+\cdots+a_{n \sigma^{\prime}(n)} \leq a_{1 \sigma(n)}+\cdots+a_{n \sigma(n)},
$$

and we find that $\pi^{\prime}:=\tau \circ \sigma^{\prime} \neq \pi$ is another minimiser. The $\operatorname{argument} \operatorname{for} \operatorname{tdet}(B)<0$ is similar.

In general, it is not true that the tropicalisation of a matrix group (relative to the standard coordinates) is a monoid under tropical multiplication.

Example 7.2 Let $G$ denote the group of $4 \times 4$-matrices of the form

$$
\left[\begin{array}{cccc}
1 & x & -x & 0 \\
0 & 1 & 0 & x \\
0 & 0 & 1 & x \\
0 & 0 & 0 & 1
\end{array}\right],
$$

where $x$ runs through the field $K$. This is a one-dimensional algebraic group isomorphic to the additive group, whose tropicalisation consists of all matrices

$$
\left[\begin{array}{cccc}
0 & a & a & \infty \\
\infty & 0 & \infty & a \\
\infty & \infty & 0 & a \\
\infty & \infty & \infty & 0
\end{array}\right]
$$

where $a \in \mathbb{R}_{\infty}$. But we have

$$
\begin{gathered}
{\left[\begin{array}{cccc}
0 & a & a & \infty \\
\infty & 0 & \infty & a \\
\infty & \infty & 0 & a \\
\infty & \infty & \infty & 0
\end{array}\right] \odot\left[\begin{array}{cccc}
0 & b & b & \infty \\
\infty & 0 & \infty & b \\
\infty & \infty & 0 & b \\
\infty & \infty & \infty & 0
\end{array}\right]} \\
=\left[\begin{array}{cccc}
0 & \min \{a, b\} & \min \{a, b\} & a+b \\
\infty & 0 & \infty & \min \{a, b\} \\
\infty & \infty & 0 & \min \{a, b\} \\
\infty & \infty & \infty & 0
\end{array}\right],
\end{gathered}
$$

which for $a, b<\infty$ does not lie in $\operatorname{Trop}(G)$.

Now consider the orthogonal group $\mathrm{O}_{n}$ consisting of all matrices $g$ that satisfy $g^{\mathrm{T}} g=I$. We do not know whether $\operatorname{Trop}\left(\mathrm{O}_{n}\right)$ is a monoid under tropical matrix multiplication, but we shall see that this tropicalisation does contain the lossy gossip monoid. For this, we take $L$ to be the field $\mathbb{C}\{\{t\}\}$ of Puiseux series in a variable $t$, and $v$ to be the order of a Puiseux series at 0 . Motivated by the analogy between the lossy phone call matrices $C_{i j}(a), a \in \mathbb{R}_{\geq 0}$ and one-parameter subgroups of algebraic groups (see Sect. 2), we introduce the one-parameter subgroups $g_{i j}(x)$ of $\mathrm{O}_{n}$ by 


$$
g_{i j}(x):=\left[\begin{array}{ccccc}
1 & & & \\
& \cos (x) & \cdots & -\sin (x) & \\
& \vdots & 1 & \vdots \\
& \sin (x) & \cdots & \cos (x) & \\
& & & & 1
\end{array}\right]
$$

where the 1's stand for identity matrices, the cosines and sines are in the $\{i, j\} \times\{i, j\}$ submatrix, and the empty entries are 0 . For any choice of $x$ in the field $L$ whose order $v(x)$ at zero is positive, the matrix $g_{i j}(x)$ is a well-defined matrix in the orthogonal group $\mathrm{O}_{n}(L)$.

Proposition 7.3 The lossy gossip monoid $G_{n}$ is contained in $\operatorname{Trop}\left(\mathrm{O}_{n}\right)$.

Proof First note that $v\left(g_{i j}(x)\right)=C_{i j}(v(x))$, so the statement would be immediate if we knew that $\operatorname{Trop}\left(\mathrm{O}_{n}\right)$ were closed under tropical matrix multiplication. We prove something weaker. Let $a_{1}, \ldots, a_{k}$ be strictly positive rational numbers and let $\left(i_{1}, j_{1}\right), \ldots,\left(i_{k}, j_{k}\right)$ be pairs of distinct indices. Then for a vector $\left(c_{1}, \ldots, c_{k}\right) \in \mathbb{C}^{k}$ outside some proper hypersurface, no cancellation takes place in the expression

$$
g_{i_{1}, j_{1}}\left(c_{1} t^{a_{1}}\right) \cdots g_{i_{k}, j_{k}}\left(c_{k} t^{a_{k}}\right)
$$

in the sense that

$$
v\left[g_{i_{1}, j_{1}}\left(c_{1} t^{a_{1}}\right) \cdots g_{i_{k}, j_{k}}\left(c_{k} t^{a_{k}}\right)\right]=v\left[g_{i_{1}, j_{1}}\left(c_{1} t^{a_{1}}\right)\right] \odot \cdots \odot v\left[g_{i_{k}, j_{k}}\left(c_{k} t^{a_{k}}\right)\right] .
$$

Here the right-hand side equals $C_{i_{1}, j_{1}}\left(a_{1}\right) \odot \cdots \odot C_{i_{k}, j_{k}}\left(a_{k}\right)$ and lies in $\operatorname{Trop}\left(O_{n}\right)$ since the left-hand side does. Since $\operatorname{Trop}\left(O_{n}\right)$ is closed in the Euclidean topology, all of $G_{n}$ is contained in it.

The dimension claim in Theorem 1.1 follows from Proposition 7.3, the BieriGroves theorem, and the fact that $\operatorname{dim} \mathrm{O}_{n}=\left(\begin{array}{l}n \\ 2\end{array}\right)$.

For $n=1,2,3$, we can say a little bit more about $\operatorname{Trop}\left(\mathrm{O}_{n}\right)$.

Example 7.4 For $n=1$, Trop $\left(\mathrm{O}_{n}\right)$ consists of the single $1 \times 1$-matrix 0 . Next, for a $2 \times 2$-matrix

$$
g=\left[\begin{array}{ll}
x & y \\
u & v
\end{array}\right] \text { with valuation }\left[\begin{array}{ll}
a & b \\
c & d
\end{array}\right]
$$

to lie in $\mathrm{O}_{2}$ we need that $x^{2}+u^{2}-1=y^{2}+v^{2}-1=0=x y+u v=0$, and these equations generate the ideal of $\mathrm{O}_{2}$. Tropicalising these equations yields that $\min \{a, c, 0\}, \min \{b, d, 0\}, \min \{a+b, c+d\}$ are all attained at least twice. This is not sufficient to characterise $\operatorname{Trop}\left(\mathrm{O}_{2}\right)$; indeed, for any negative $a, b$ the matrix

$$
\left[\begin{array}{ll}
a & b \\
a & b
\end{array}\right]
$$


satisfies all tropical equations above, but (unless $a=b$ ) not the tropicalisation of the equation $x v-y u=1$ which expresses that $\mathrm{O}_{2} \subseteq \mathrm{SL}_{2}$. Imposing this additional condition, i.e. that $\min \{a+d, b+c, 0\}$ is attained at least twice, we find that $\operatorname{Trop}\left(\mathrm{O}_{2}\right)$ consists of three cones:

$$
\begin{aligned}
\operatorname{Trop}\left(\mathrm{O}_{2}\right)= & \left\{\left[\begin{array}{ll}
0 & a \\
a & 0
\end{array}\right] \mid a \in[0, \infty]\right\} \cup\left\{\left[\begin{array}{ll}
a & 0 \\
0 & a
\end{array}\right] \mid a \in[0, \infty]\right\} \\
& \cup\left\{\left[\begin{array}{ll}
a & a \\
a & a
\end{array}\right] \mid a \in(-\infty, 0]\right\} .
\end{aligned}
$$

The first cone is $G_{2}$, the second cone is $G_{2}$ with the columns reversed, and the third cone makes the fan balanced.

In general, if a variety is stable under a coordinate permutation, then its tropicalisation is stable under the same coordinate permutation. Consequently, $\operatorname{Trop}\left(O_{n}\right)$ is stable under permuting rows, under permuting columns, and under matrix transposition.

For $n=3$, a computation using gfan [15] shows that the quadratic equations expressing that columns and rows both form orthonormal bases, together with the equation det -1 , do not form a tropical basis. For example, the four-dimensional cone of matrices

$$
\left[\begin{array}{lll}
a & a & b \\
a & a & b \\
c & c & d
\end{array}\right]
$$

with $a \leq b \leq c \leq 0 \leq d$ is contained in the tropical prevariety defined by the corresponding tropical equations, and for dimension reasons cannot belong to the three-dimensional fan $\operatorname{Trop}\left(\mathrm{O}_{3}\right)$.

However, these quadratic equations do suffice to prove that $\operatorname{Trop}\left(\mathrm{O}_{3}\right) \cap[0, \infty]^{3 \times 3}$ is equal to $\operatorname{Sym}(3) \cdot G_{3}$, i.e. obtained from $G_{3}$ by permuting rows. Indeed, let a $3 \times 3$ matrix $A$ in $[0, \infty]^{3 \times 3}$ satisfy the tropicalisations of these equations. Then $\operatorname{tdet}(A)=0$, hence after permuting rows $A$ has zeroes on the diagonal. Now we distinguish two cases. First, assume that $A$ is symmetric:

$$
A=\left[\begin{array}{lll}
0 & a & b \\
a & 0 & c \\
b & c & 0
\end{array}\right] .
$$

Then we claim that $A$ lies in $D_{3}$. Indeed, suppose that $a>b+c$. Then the tropicalisation of the condition that the first two columns are perpendicular does not hold for $A$. Hence $a \leq b+c$ and similarly for the other triangle inequalities; we conclude that $A \in D_{3}$. Next, assume that $A$ is not symmetric. After conjugation with a permutation matrix, we may assume that $A$ is of the form

$$
\left[\begin{array}{lll}
0 & a & b \\
d & 0 & c \\
e & f & 0
\end{array}\right]
$$


with $a>d$. Then the tropical perpendicularity of the first two columns yields $d=e+f$, that of the last two columns yields $c=f$, and that of the first two rows yields $d=b+c$. So $A$ looks like

$$
\left[\begin{array}{ccc}
0 & a & b \\
b+c & 0 & c \\
b & c & 0
\end{array}\right]
$$

which is one of the cones in $G_{3}$.

Remark 7.5 We do not know whether the equality $\operatorname{Sym}(n) \cdot G_{n}=\operatorname{Trop}\left(\mathrm{O}_{n}\right) \cap$ $[0, \infty]^{n \times n}$ (where the action of $\operatorname{Sym}(n)$ is by left multiplication) holds for all $n$. If true, then this would be interesting from the perspective of algebraic groups over non-Archimedean fields: it would say that the image under $v$ of the compact subgroup $\mathrm{O}_{n}\left(L^{0}\right) \subseteq \mathrm{O}_{n}(L)$, where $L^{0}$ is the valuation ring of $L$, is (dense in) the lossy gossip monoid. But we see no reason to believe that this is true in general. A computational hurdle to checking this even for $n=4$ is the computation of a polyhedral fan supporting Trop $\left(\mathrm{O}_{n}\right)$. For $n=3$ this can still be done using $g$ fan, and it results in a fan with $f$-vector $(580,1698,1143)$. Among the 1143 three-dimensional cones, 1008 are contained in the positive orthant, as opposed to the $6 \cdot 7=42$ found by applying row permutations to the cones in $G_{3}$. This suggests that $g$ fan does not automatically find the most efficient fan structure on $\mathrm{O}_{n}$, and at present we do not know how to overcome this.

\section{Ordinary Gossip}

In this section we study the ordinary gossip monoid $G_{n}(\{0, \infty\})$, which is the submonoid of $G_{n}$ of matrices with entries in $\{0, \infty\}$. Note that there is a surjective homomorphism $G_{n} \rightarrow G_{n}(\{0, \infty\})$ mapping non- $\infty$ entries to 0 and $\infty$ to $\infty$, which shows that the length of an element of $G_{n}(\{0, \infty\})$ inside $G_{n}$ is the same as the minimal number of non-lossy phone calls $C_{i j}(0)$ needed to express it. A classical result says that length of the all-zero matrix is exactly 1 for $n=2,3$ for $n=3$, and $2 n-4$ for $n \geq 4$ $[1,6,12,19]$, and this result spurred a lot of further activity on gossip networks. But the all-zero matrix does not necessarily have the largest possible length—see Table 2,

Table 2 Sizes and maximal lengths of $G_{n}(\{0, \infty\})$ for $n=1, \ldots, 9$

\begin{tabular}{llc}
\hline$n$ & $\left|G_{n}(\{0, \infty\})\right|$ & Max. length \\
\hline 1 & 1 & 0 \\
2 & 2 & 1 \\
3 & 11 & 3 \\
4 & 189 & 4 \\
5 & 9152 & 6 \\
6 & $1,092,473$ & 10 \\
7 & $293,656,554$ & 13 \\
8 & $166,244,338,221$ & 16 \\
9 & $188,620,758,836,916$ & 19 \\
\hline
\end{tabular}


which records sizes and maximal element lengths for $G_{n}(\{0, \infty\})$ with $n \leq 9$. The first 8 rows were computed by former Eindhoven Master's student Jochem Berndsen [3].

While we do not know the maximal length of an element in $G_{n}(\{0, \infty\})$ for general $n$, we do have an upper bound, namely, the maximal number of factors in an irredundant product. This number, in turn, is bounded from above by $\left(\begin{array}{l}n \\ 2\end{array}\right)$, as we now prove.

Proof of Theorem 1.4 and Corollary 1.5 Consider $n$ gossipers, initially each with a different gossip item unknown to all other gossipers. They communicate by telephone, and whenever two gossipers talk, each tells the other all he knows. We will determine the maximal length of a sequence of calls, when in each call at least one participant learns something new. The answer turns out to be $\left(\begin{array}{l}n \\ 2\end{array}\right)$.

That $\left(\begin{array}{l}n \\ 2\end{array}\right)$ is a lower bound is shown by the following scenario: Number the gossipers $1, \ldots, n$. All calls involve gossiper 1 . For $i=2, \ldots, n$ he calls $i, i-1, \ldots, 2$, for a total of $1+2+\cdots+(n-1)=\left(\begin{array}{l}n \\ 2\end{array}\right)$ calls. There are many other scenarios attaining $\left(\begin{array}{l}n \\ 2\end{array}\right)$, and it does not seem easy to classify them.

We now argue that $\left(\begin{array}{l}n \\ 2\end{array}\right)$ is an upper bound. Although we will not use this, we remark that it is easy to see that $2 \cdot\left(\begin{array}{l}n \\ 2\end{array}\right)=n(n-1)$ is an upper bound. After all, each of the $n$ participants must learn $n-1$ items, and in each call at least one participant learns something.

Let $I_{1}, I_{2}, \ldots, I_{\ell}$ be a sequence of unordered pairs from $[n]$ representing phone calls where in each call at least one participant learns something new. To each $I_{a}$ we associate the homomorphism $\phi_{a}:=\mathrm{SO}_{2}(\mathbb{C}) \rightarrow \mathrm{SO}_{n}(\mathbb{C})$ that maps a $2 \times 2$-matrix $g$ to the matrix that has $g$ in the $I_{a} \times I_{a}$-block and otherwise has zeroes outside the diagonal and ones on the diagonal. For each $k \leq \ell$ we obtain a morphism of varieties (not a group homomorphism) $\psi_{k}: \mathrm{SO}_{2}(\mathbb{C})^{k} \rightarrow \mathrm{SO}_{n}(\mathbb{C})$ sending $\left(g_{1}, \ldots, g_{k}\right)$ to $\phi_{1}\left(g_{1}\right) \cdots \phi_{k}\left(g_{k}\right)$. Let $X_{k}$ be the closure of the image of $\psi_{k}$; this is an irreducible subvariety of $\mathrm{SO}_{n}(\mathbb{C})$. The $(i, j)$-matrix entry is identically zero on $X_{k}$ if and only if gossiper $j$ does not know gossip $i$ after the first $k$ phone calls. Since some gossiper learns something new in the $k$-th phone call, some matrix entry is identically zero on $X_{k-1}$ which is not identically zero on $X_{k}$. Consequently, we have $0=\operatorname{dim} X_{0}<\operatorname{dim} X_{1}<\cdots<\operatorname{dim} X_{\ell}$. But all $X_{k}$ are contained in the variety $\mathrm{SO}_{n}(\mathbb{C})$ of dimension $\left(\begin{array}{l}n \\ 2\end{array}\right)$, so we conclude that $\ell \leq\left(\begin{array}{l}n \\ 2\end{array}\right)$.

This concludes the proof of Theorem 1.4. Corollary 1.5 follows because, in any irredundant product of phone calls, every initial segment must be a sequence of phone calls in each of which at least one party learns something new.

We computed the longest irredundant products of phone calls for small $n$, see Table 3.

Table 3 Maximum length $l_{n}$ of an irredundant product of phone calls

\begin{tabular}{lllllllll}
\hline$n$ & 1 & 2 & 3 & 4 & 5 & 6 & 7 & 8 \\
\hline$l_{n}$ & 0 & 1 & 3 & 5 & 8 & 12 & 16 & $\geq 21$ \\
\hline
\end{tabular}




\section{Open Questions}

In view of the extensive computations in Sects. 4-6 and the rather indirect dimension argument in Sect. 7, the most urgent challenge concerning the lossy gossip monoid is the following.

Question 9.1 Find a purely combinatorial description of a polyhedral fan structure with support $G_{n}$. Use this description to prove or disprove the pureness of dimension $\left(\begin{array}{l}n \\ 2\end{array}\right)$ and the connectedness in codimension one.

The following question is motivated on the one hand by the fact that $G_{n}$ has dimension $\left(\begin{array}{l}n \\ 2\end{array}\right)$ and on the other hand by Theorem 1.4, which implies that elements of the ordinary gossip monoid $G_{n}(\{0, \infty\})$ have length at most $\left(\begin{array}{l}n \\ 2\end{array}\right)$.

Question 9.2 Is the length of any element of $G_{n}$ at most $\left(\begin{array}{l}n \\ 2\end{array}\right)$ ?

Once a satisfactory polyhedral fan for $G_{n}$ is found, the somewhat ad hoc graphs in Sects. 4 and 5 lead to the following challenge.

Question 9.3 Find a useful notion of optimal realisations of elements of $G_{n}$ by graphs with detours, and a notion of tight spans of such elements.

For the relation between tight spans and optimal realisations of metrics by weighted graphs see [8, Thm. 5].

We conclude with two questions concerning tropicalisations of orthogonal groups (Sect. 7).

Question 9.4 Is $\operatorname{Trop}\left(\mathrm{O}_{n}\right)$ a monoid under tropical matrix multiplication? This is evident for $n \leq 2$, we have checked it computationally for $n=3$, and it is open for $n \geq 4$.

Question 9.5 Is it true that $\operatorname{Trop}\left(\mathrm{O}_{n}\right) \cap[0, \infty]^{n \times n}$ equals $\operatorname{Sym}(n) \cdot G_{n}$ ? Here the action of $\operatorname{Sym}(n)$ is by permuting rows. This is true for $n \leq 3$, and open for $n \geq 4$.

Acknowledgments We thank Tyrrell McAllister for discussions on the tropical orthogonal group many years ago, and Peter Fenner and Mark Kambites for pointing out problems with an earlier, purely combinatorial proof of Theorem 1.4. JD is supported by a Vidi Grant from the Netherlands Organisation for Scientific Research (NWO) and BJF by an NWO free competition Grant.

Open Access This article is distributed under the terms of the Creative Commons Attribution License which permits any use, distribution, and reproduction in any medium, provided the original author(s) and the source are credited.

\section{References}

1. Baker, B., Shostak, R.: Gossips and telephones. Discrete Math. 2, 191-193 (1972)

2. Berman, K.A., Hawrylycz, M.: Telephone problems with failures. SIAM J. Algebraic Discrete Methods 7, 13-17 (1986)

3. Berndsen, J.: Three problems in algebraic combinatorics. Master's thesis, Eindhoven University of Technology (2012). http://alexandria.tue.nl/extra1/afstversl/wsk-i/berndsen2012 
4. Bieri, R., Groves, J.R.J.: The geometry of the set of characters induced by valuations. J. Reine Angew. Math. 347, 168-195 (1984)

5. Boyd, S., Ghosh, A., Prabhakar, B., Shah, D.: Randomized gossip algorithms. IEEE Trans. Inform. Theory 52, 2508-2530 (2006)

6. Bumby, R.T.: A problem with telephones. SIAM J. Algebraic Discrete Methods 2, 13-18 (1981)

7. Butkovič, P.: Max-Linear Systems, Theory and Algorithms. Springer Monographs in Mathematics. Springer, London (2010)

8. Dress, A.W.M.: Trees, tight extensions of metric spaces, and the cohomological dimension of certain groups: a note on combinatorial properties of metric spaces. Adv. Math. 53, 321-402 (1984)

9. Dress, A., Huber, K.T., Lesser, A., Moulton, V.: Hereditarily optimal realizations of consistent metrics. Ann. Combin. 10(1), 63-76 (2006)

10. Gawrilow, E., Joswig, M.: Polymake: a framework for analyzing convex polytopes. In: Kalai, G., Ziegler, G.M. (eds.) Polytopes-Combinatorics and Computation. DMV Seminars, vol. 29, pp. 43-74. Birkhäuser, Basel (2000)

11. Haddad, R.W., Roy, S., Schäffer, A.A.: On gossiping with faulty telephone lines. SIAM J. Algebr. Discrete Methods 8, 439-445 (1987)

12. Hajnal, A., Milner, E.C., Szemerédi, E.: A cure for the telephone disease. Can. Math. Bull. 15, 447-450 (1972)

13. Imrich, W., Simões Pereira, J.M.S., Zamfirescu, C.M.: On optimal embeddings of metrics in graphs. J. Combin. Theory Ser. B 36(1), 1-15 (1984)

14. Izhakian, Z., Johnson, M., Kambites, M.: Tropical matrix groups (2012). http://arxiv.org/abs/1203. 2449

15. Jensen, A.N.: Gfan, a software system for Gröbner fans and tropical varieties. http://home.imf.au.dk/ jensen/software/gfan/gfan.html (2005-2011)

16. Koolen, J., Lesser, A., Moulton, V.: Optimal realizations of generic five-point metrics. Eur. J. Combin. 30(5), 1164-1171 (2009)

17. Maclagan, D., Sturmfels, B.: Introduction to Tropical Geometry. Graduate Studies in Mathematics, vol. 161. American Mathematical Society, Providence, RI (2015)

18. Sturmfels, B., Yu, J.: Classification of six-point metrics. Electron. J. Combin. 11(1), R44 (2004)

19. Tijdeman, R.: On a telephone problem. Nieuw Arch. Wiskd. III Ser. 19, 188-192 (1971)

20. Ziegler, G.M.: Lectures on Polytopes. Graduate Texts in Mathematics, vol. 152. Springer, Berlin (1995) 